\title{
Light transmission assisted by Brewster-Zennek modes in chromium films carrying a subwavelength hole array
}

\author{
Michaël Sarrazin $*$ and Jean-Pol Vigneron \\ Laboratoire de Physique du Solide, \\ Facultés Universitaires Notre-Dame de la Paix, \\ rue de Bruxelles 61, B-5000 Namur, Belgium
}

(Dated: November 19, 2018)

\begin{abstract}
This work confirms that not only surface plasmons but many other kinds of electromagnetic eigenmodes should be considered in explaining the values of the transmittivity through a slab bearing a two-dimensional periodic corrugation. Specifically, the role of Brewster-Zennek modes appearing in metallic films exhibiting regions of weak positive dielectric constant. It is proposed that these modes play a significant role in the light transmission in a thin chromium film perforated with normal cylindrical holes, for appropriate lattice parameters.

PACS numbers: 78.20.-e, 42.79.Dj, 42.25.Bs, 42.25.Gy
\end{abstract}

\section{INTRODUCTION}

The optical properties of thin metallic films containing periodic arrays of subwavelength holes have been actively studied in relation with the possibility of transmission engineering. Originally, the remarkable optical transmission found in these structures was pointed out by T.W. Ebbesen et al back in 1998 ${ }^{1}$. After these early experimental observations, the role of the thin metallic film surface plasmons (SPs) was put forward in order to explain the peculiar wavelength dependence of the transmission 1.2.3.4.5.6.7.8.9. As a consequence of this suggestion, it was generally believed that the typical transmission features could only be obtained with metallic films ${ }^{1.6}$. However it is important to note that other explanations had been developed which could compete with the SPs mode $1^{9.10 .11 .12 .13 .14}$. For instance, it was suggested that these phenomena could also be described in terms of the short range diffraction of evanescent waves 10 , or in terms of dynamical diffraction effects 11 . Another explanation suggests to emphasize the role of cavity resonances taking place in the holes to explain the transmission enhancement $\stackrel{9}{ }$.

Many questions remain to completely clarify the scattering processes involved in these experiments. Nevertheless, even if the exact role of SPs is not clearly assessed $^{1,2.3 .4 .5 .6 .7 .8 .9}$, most interpretations tend to admit that SPs should play a key role in the observed transmission spectrum features. The observed transmission exhibits a set of peaks and dips. Many authors suggested that transmission peaks are created by SPs resonances $1,2,3,4,5,6,7.8$ but others rather saw the SPs resonances at the spectral dips ${ }^{9}$. In a recent work ${ }^{13}$ we suggested to redefine the role of SPs in Ebbesen experiments in the required context of resonant Wood anomalies ${ }^{15}$. In doing so, we have shown that the transmission spectrum could be better depicted as a series of Fano profiles $\underline{16}$.

FIG. 1: A view of the system under study
These recognizable lineshapes result from the interference of non resonant transfers with resonant transfers which involve the film SPs and evanescent diffraction orders. We could then point out that each transmission peak-dip pair is nothing else than a Fano profile $\frac{16}{}$. The appearance of an assymetric Fano lineshape does not necessarily locates the peak or the dip at the SP resonance $\frac{13}{}$. However, we have shown that the existence of the SPs is a condition for the presence of the Fano lineshapes 13 . It can be noted that recent results by C. Genet et al confirm this description ${ }^{14}$.

As a further outcome of this work ${ }^{13}$ it became clear this kind of transmission spectrum, with its Fano lineshapes, could also be obtained in a more general context and that the generic concept of eigenmodes could substitute that of SPs. These results implied two important new ideas. First, according to the contributions of resonant and nonresonant processes in the Fano profile, eigenmodes can be associated with wavelengths closer to the peaks or to the dips. Second, it is possible to obtain transmission curves similar to those of metal films, by substituting SPs with guided modes or other types of polaritons. Many examples can be found, including highly refractive materials defining guided modes or ionic crystals in the restrahlen band defining phonon-polaritons.

In a previous paper ${ }^{13}$, it was made clear that a strong hypothesis on the origin of the eigenmodes was not required to account for the general features of the membrane transmission. As metal films were considered there, it seemed natural to involve SPs in the transmission mechanisms. By contrast, in another recent paper ${ }^{17}$, simulations of light transmission through an array of subwavelength cylindrical holes in a tungsten layer deposited on glass substrate was given an interpretation based on dielectric guided modes. Indeed, in the wavelength domain under examination, tungsten exhibits a positive permittivity real part $\frac{18}{}$. Though SPs cannot exist, the transmission pattern is found very similar to that obtained with a metallic film. These theoretical results have recently been confirmed experimentally $\underset{12}{ }$. Surprisingly, the very fact that the typical transmission pattern can 
FIG. 2: Real $\left(\varepsilon_{m}^{\prime}\right)$ and imaginary $\left(\varepsilon_{m}^{\prime \prime}\right)$ part of the chromium permittivity as a function of wavelength

be observed even in non-metallic systems convinced some authors 12 to fully reject the SPs hypothesis and rather consider models involving non-resonant evanescent waves diffraction. To our knowledge, this point of view is not supported by recent results ${ }^{13,14,17}$ and the SPs must be replaced by other kinds of eigenmodes ${ }^{13.17}$ in the nonmetallic cases.

The purpose of the present paper is to demonstrate that the transmission profile of a chromium film, in the restricted wavelength domain $(1112-1292 \mathrm{~nm})$ where the dielectric constant is positive ${ }^{18}$, should involve eigenmodes which are not SPs nor guided modes. In this case, SPs can be substituted by Brewster-Zennek modes (BZ modes). This confirms that the observation of the effects described by T.W. Ebbesen et al in systems where SPS do not exist do not preclude the existence of a mechanism involving the excitation of other eigenmodes.

\section{BREWSTER-ZENNEK MODES}

Let us recall some properties of surface modes, including the concept of BZ modes ${ }^{19,20}$. We consider an interface between two media, 1 and 2, described by their respective permittivities $\varepsilon_{1}$ and $\varepsilon_{2}$. It is well know that for an isotropic material, only $p$ polarized modes can occur as a surface modes. We define $k$ as the wave vector component parallel to the interface and we set $k_{0}=\omega / c$, where $\omega$ is the angular frequency of the mode. Then, for the surface mode, the normal component of the wave vector is given in each medium by

$$
\beta_{i}=\sqrt{k^{2}-k_{0}^{2} \varepsilon_{i}}
$$

The subscript $i$ denotes one of the media 1 or 2 .

In the following we enforce a real positive value of $\varepsilon_{1}$, which means that medium 1 is a lossless dielectric material, while we admit the form $\varepsilon_{2}=\varepsilon_{2}^{\prime}+i \varepsilon_{2}^{\prime \prime}\left(\varepsilon_{2}^{\prime \prime} \geq 0\right)$ for medium 2, which means that this medium is allowed to generate losses. Applying electromagnetic boundary conditions pertinent to complex-response discontinuities, we are lead to the following well-known expression which controls the appearance of surface modes 21

$$
\varepsilon_{1} \beta_{2}+\varepsilon_{2} \beta_{1}=0
$$

From this, and eq. (1), we can develop the following expression, out of which a dispersion relation can be drawn

$$
k^{2}=k_{0}^{2} \frac{\varepsilon_{1} \varepsilon_{2}}{\varepsilon_{1}+\varepsilon_{2}}
$$

However, it is important to realize that in this expression, the factors $\beta_{i}$ have been squared, so that eq. (3) and (2) are not equivalent. The solutions of eq. (2) can be found among those of eq. (3), but some of the solutions of eq. (3) are spurious and should be discarded. One way to do this is to take the possible values of $k^{2}$ from eq. (3), inject them in eq. (2), and check for the correct matching. This actually generates a second condition, which can be used together with eq. (3), to make precise the acceptable eigenmodes branches. This condition turns out to be

$$
\sqrt{\frac{-z^{2}}{1+z}}+z \sqrt{\frac{-1}{1+z}}=0
$$

where, somewhat surprisingly, the only variable that needs to be examined is the ratio $z=\varepsilon_{2} / \varepsilon_{1}$. The function of $z$, at the left-hand side of this equation, when carefully calculated, defines a border line which splits the complex plane into two extended connected regions. On one side of this border, the left-hand side of eq. (4) cannot vanish, while on the other side, it will vanish everywhere. The surface states can only show up when the ratio $z$ lies in this latter region.

The border separating the two regions can be obtained as follows. Both terms added in eq. 4 are complex numbers with the same modulus. In one of the regions mentioned above, they are opposite complex numbers and they cancel each other; in the rest of the complex plane, they turn out to be identical and they cannot add to zero. The borderline will then be found at the complex points $z$ where the sign determination of one of the square roots in eq. 4 changes, and this requires to look for the cut of the complex square-root function $\sqrt{Z}$. It is convenient to take this cut as the straight segment $Z=-i \gamma(\gamma$ real and positive) and this choice lead us to identify two distinct implicit paths,

$$
-\frac{1}{1+z}=-i \gamma
$$

and

$$
-\frac{z^{2}}{1+z}=-i \gamma
$$

which form the border. This choice of the cut is dictated in part by the choice of a time-dependent oscillation written as $\exp (-i \omega t)$ and the requirement that $\sqrt{1}=1$. Once the borderline is known, a quick check at the case $z=1$ shows that no cancellation can occur there, so that the active region where eigenmodes appear is, unambiguously, that region which does not contain $z=1$.

On the basis of this analysis, it is easily shown that, whatever $\varepsilon_{2}=\varepsilon_{2}^{\prime}+i \varepsilon_{2}^{\prime \prime}$ such that $\varepsilon_{2}^{\prime}<0$ and $\varepsilon_{2}^{\prime \prime} \geq 0$ surface eigenmodes exist. These modes are SPs and they will appear at metal/dielectric interfaces regardless of the metal component of the heterojunction.

If $\varepsilon_{2}^{\prime} \geq 0$, surface eigenmodes exist only when $0 \leq \varepsilon_{2}^{\prime}<$ $\varepsilon_{1}$ if the condition

$$
\varepsilon_{2}^{\prime \prime}>\varepsilon_{2}^{\prime} \sqrt{\frac{\varepsilon_{1}+\varepsilon_{2}^{\prime}}{\varepsilon_{1}-\varepsilon_{2}^{\prime}}}
$$


FIG. 3: (Color online) Comparison between transmission and resonances. Solid line : $a=1000 \mathrm{~nm}$, Dashed line : $a=1200$ nm. (a) : zeroth order transmission against wavelength. A and $\mathrm{B}$ are the main maxima of transmission. (b) : Resonant diffraction orders amplitudes related to the vectors $(0, \pm 1)$ of the reciprocal lattice, at the substrate/metal interface (1) and vaccuum/metal interface (2), as a function of the wavelength

is fulfilled. Obviously such modes are not SPs and it is well known that a dielectric/dielectric interface, without loss, cannot support a surface mode if $\beta_{i}$ is real. Nevertheless, surface modes can exist for a dielectric/dielectric interface if one of the media is a lossy material such that the imaginary part of its permittivity verify the above conditions. Such modes are known as Brewster-Zenneck modes. They verify $\operatorname{Re}\{k\}<\frac{\omega}{c} \sqrt{\varepsilon_{1}}$ and are necessarily related, by definition, to radiative surface modes (contrasting SPs in metals such that iron, gold or copper). Fundamentally it is well known that SPs consist in a collective motion of electrons ${ }^{21}$. In the context of an electromagnetic model, the electronic character of SPs is then contained in the permittivity $\varepsilon_{2}$. Indeed, the permittivity properties take their origin in the electronic characteristics of the medium ${ }^{21}$. This is true whatever the value of $\varepsilon_{2}^{\prime}$, positive or negative. So, despite the above-mentioned differences between SPs and BZ modes, both are related intrinsically to a collective electronic phenomenon. In this way, the transition from the SPs towards the BZ modes and vice versa occurs without discontinuities. Note that the present paper deals with metals only. In the case of ionic crystals in the far infrared, it would be natural to expect for the BZ modes counterpart associated with phonon-polaritons instead of SPs.

\section{BREWSTER-ZENNEK-BASED TRANSMISSION}

Now we study the properties of the surface modes in the case of a chromium grating similar to those submitted to experiments by Thio et $a \underline{\underline{\underline{3}}}$ (see fig. (1). In a large part of the spectrum of interest here, the real part of the chromium permittivity is negative (fig. 2), as for simple metals below the plasma frequency. However, for wavelengths in the interval from 1112 to $1292 \mathrm{~nm}$, it becomes positive, as, specifically, we observe $0<\varepsilon_{2}^{\prime}<0,4$ and $25,54<\varepsilon_{2}^{\prime \prime}<27,3$. With vacuum and substrate (glass) permittivities equal to 1 and 2,24 respectively, the conditions for the formation of $\mathrm{BZ}$ modes are verified at both interfaces of the chromium film. This is a peculiar situation : with tungsten films in the dielectric domain ${ }^{17.18}$, for instance, the same conditions are not fulfilled and the BZ modes do not appear.

The transmission spectrum can easily be understood if we first localize the BZ modes in the spectrum of a flat, homogeneous film. For normal incident light falling on a such a homogeneous film considered periodic on a square lattice of parameter $a$ (the "empty" lattice case), surface modes resonance will appear at the wavelength $\lambda_{i, j}$ provided by the following equation ${ }^{21}$

$$
\lambda_{i, j}=\frac{a}{\left(i^{2}+j^{2}\right)^{1 / 2}} \operatorname{Re} \sqrt{\frac{\varepsilon_{1} \varepsilon_{2}}{\varepsilon_{1}+\varepsilon_{2}}}
$$

where $(i, j)$ denotes the related vector $\mathbf{g}$ of the reciprocal lattice, such that $\mathbf{g}=(2 \pi / a)(i, j)$. These wavelengths are indicative only, as they are based on a model where the cylindrical holes have not been accounted for.

More detailed simulations which do not contain this simplification have been carried out. The calculations which will now be described are based on a coupledmodes method which combines a scattering matrix formalism with a plane wave representation of the fields. This technique provides a computation scheme for the amplitude and polarization ( $s$ or $p$ ) of reflected and transmitted fields in any diffracted order. A brief account of the method has been presented elsewhere $\frac{13.22 .23}{}$ so that we will not recall any technicality about these computations.

Following the experiments ${ }^{3}$, fig. 3 shows the calculated zeroth order transmission of a grating prepared by perforating a $100 \mathrm{~nm}$ thick chromium film deposited on glass with circular holes of $250 \mathrm{~nm}$ radius (fig. 1). The two-dimensional square lattice parameter is $a=1000 \mathrm{~nm}$ (solid line) or $a=1200 \mathrm{~nm}$ (dashed line). The incident beam is normal to the grating surface and polarized so that the electromagnetic wave electric field is oriented along one of the holes nearest-neighbor directions $(O y$ axis). A and B on Fig. B indicate the main transmission maxima. Fig. $3 \mathrm{~b}$ represents the amplitude of the calculated resonant $p$-polarized diffraction orders $(0, \pm 1)$ for the substrate/metal interface (peak 1) and for the vacuum/metal interface (peak 2). Again, in Fig. 3b, the dashed line refers to a lattice parameter of $a=1200 \mathrm{~nm}$. It can be noted that, as justified by the above discussion, these peaks do not correspond to the transmission maxima. We have shown that the spectral lineshape of the resonance can be interpreted as a Fano's profile13.14. What is remarkable here is that, for a lattice parameter of $a=1200 \mathrm{~nm}$, the resonance has moved to a spectral region $(1200 \mathrm{~nm})$ where the real part of the chromium permittivity is positive, and that clearly SPs do not exist there. In this situation the resonance is related to the appearance of a BZ mode. Fig 4 shows the location of both peaks (1) and (2) as a function of the grating parameter $a$ (solid lines (1) and (2), respectively). We could compare these values with the wavelengths obtained from eq. 8 (dashed lines (1) and (2) respectively) for the appropriate diffraction orders. A good agreement is found between these calculated quantities. We show that as the lattice parameter increases, the resonance wavelength increases linearly as predicted by eq. 8 The hatched domain in fig. 4 is characterized by the presence of BZ modes. The SP domain lies everywhere else. One interesting observation is that both kinds of surface modes (BZ modes and 
FIG. 4: Position of both peaks (1) and (2) against grating parameters $a$. Solid lines (1) and (2) respectively are obtained from numerical computations. Dashed lines (1) and (2) are obtained from eq.(8). A (circle dots) and B (square dots) : location of both main maxima of transmission against $a$. Hatched domain : BZ modes, elsewhere : SPs modes.

SPs modes) substitute each others in a continuous way in accordance with our previous explanation in section II. The location of transmission maxima A (circle dots) and B (square dots) are also shown on fig. 4 These, as already underlined, do not indicate directly the eigenmodes resonance wavelengths though these transmission maxima are known to be related to the surface modes via a Fano profile.

Note that, in a previous work ${ }^{4}$, it was inferred that the SPs wavelength were red-shifted, compared to those calculated for an empty lattice. It was assumed that such a shift was needed to make the transmission maxima coincide with SPs wavelengths and the justification suggested for this shift was based on the presence of holes. This is not observed in actual detailed calculations. The present results show that the exact resonant wavelengths of the surface modes, as computed numerically, are not shifted enough to justify their location on transmission maxima. Nevertheless, as mentioned before, an eigenmode resonance does not necessarily coincide with a transmission maximum, but a maximum-minimum pair indicates the presence of a nearby eigenmode resonance ${ }^{13}$. In fact, in this case, the surface modes resonances are incidentally located closer to the minima of the transmission. It is interesting to note that Thio et al have compared experimentally the transmission from gratings similar to ours, with lattice parameters $a=1000 \mathrm{~nm}$ and $a=1200 \mathrm{~nm}^{3}$. The present computations of the transmission matched very well with these data 3 . We take this as a strong evidence of the relevance of the Brewster-Zennek modes in the description of these experiments.

\section{CONCLUSION}

In the context of studies of the optical transmission properties of subwavelength hole arrays, we have shown that Brewster-Zennek modes can substitude surface plasmons when considering a layer made from a lossy dielectric medium, for specific lattice parameters. When modelling a grating etched in a chromium film we can use the concept of Brewster-Zennek modes to explain the spectra found in previous experiments. This study recalls the existence requirement of eigenmodes for observing the typical transmission lineshapes and we have shown that, contrasting a frequent believe, surface modes wavelengths based on an empty lattice model are not significatively red-shifted to justify their location on transmission maxima.

\section{Acknowledgments}

We acknowledge the use of the Namur Interuniversity Scientific Computing Facility (Namur-ISCF), a joint project between the Belgian National Fund for Scientific Research (FNRS), and the Facultés Universitaires NotreDame de la Paix (FUNDP).

This work was partially supported by the EU BelgianFrench INTERREG III project "PREMIO", the EU5 Centre of Excellence ICAI-CT-2000-70029 and the InterUniversity Attraction Pole (IUAP P5/1) on "Quantumsize effects in nanostructured materials" of the Belgian Office for Scientific, Technical, and Cultural Affairs.
* Electronic address: michael.sarrazin@fundp.ac.be

1 T.W. Ebbesen, H.J. Lezec, H.F. Ghaemi, T. Thio, P.A. Wolff, Nature (London) 391, 667 (1998).

${ }^{2}$ U. Schröter, D. Heitmann, Phys. Rev. B 58, 15419 (1998).

3 T. Thio, H.F. Ghaemi, H.J. Lezec, P.A. Wolff, T.W. Ebbesen, JOSA B 16, 1743 (1999).

${ }^{4}$ H.F. Ghaemi, T. Thio, D.E. Grupp, T.W. Ebbesen, H.J. Lezec, Phys. Rev. B 58, 6779 (1998).

5 J.A. Porto, F.J. Garcia-Vidal, J.B. Pendry, Phys. Rev. Lett. 83, 2845 (1999).

6 D.E. Grupp, H.J. Lezec, T.W. Ebbesen, K.M. Pellerin, T. Thio, Appl. Phys. Lett. 77, 1569 (2000).

7 T. Thio, H.J. Lezec, T.W. Ebbesen, Physica B 279, 90 (2000).

8 A. Krishnan, T. Thio, T. Kim, H. Lezec, T. Ebbesen, P. Wolff, J.B.Pendrya, L. Martin-Moreno, and F. GarciaVidal, Opt. Commun. 200, 1 (2001).

9 Q. Cao, Ph. Lalanne, Phys. Rev. Lett. 88, 057403 (2002).

10 J.-M. Vigoureux, Optics Comm. 198, 257 (2001).

11 M.M.J. Treacy, Phys. Rev. B 66, 195105 (2002).
12 H.J. Lezec, T. Thio, Optics Express 12, 3629 (2004).

13 M. Sarrazin, J.-P. Vigneron, J.-M. Vigoureux, Phys. Rev. B 67, 085415 (2003).

14 C. Genet, M. P. van Exter and J. P. Woerdman, Optics Comm. 225, 331 (2003).

15 R. Wood, Phys. Rev. 48, 928 (1935).

16 V. Fano, Ann. Phys. 32, 393 (1938).

17 M. Sarrazin, J.-P. Vigneron, Phys. Rev. E 68, 016603 (2003).

18 D.W. Lynch, W.R. Hunter, in Handbook of Optical Constants of Solids, edited by E. D. Palik (Academic Press, Inc., 1991), vol. 2.

19 G. S. Agarwal, Phys. Rev. B 8, 4768 (1973).

20 F. Yang, J.R. Sambles, G.W. Bradberry, Phys. Rev. B 44, 5855 (1991).

21 H. Raether, in Springer Tracts in Modern Physics, edited by G. Hohler, E.A. Nickisch (Springer, Berlin, 1988), vol. 111.

22 M. Sarrazin, J.-P. Vigneron, Optics Commun. 240, 89 (2004). 
23 J.P. Vigneron, F. Forati, D. André, A. Castiaux, I. Derycke, A. Dereux, Ultramicroscopy 61, 21 (1995). 


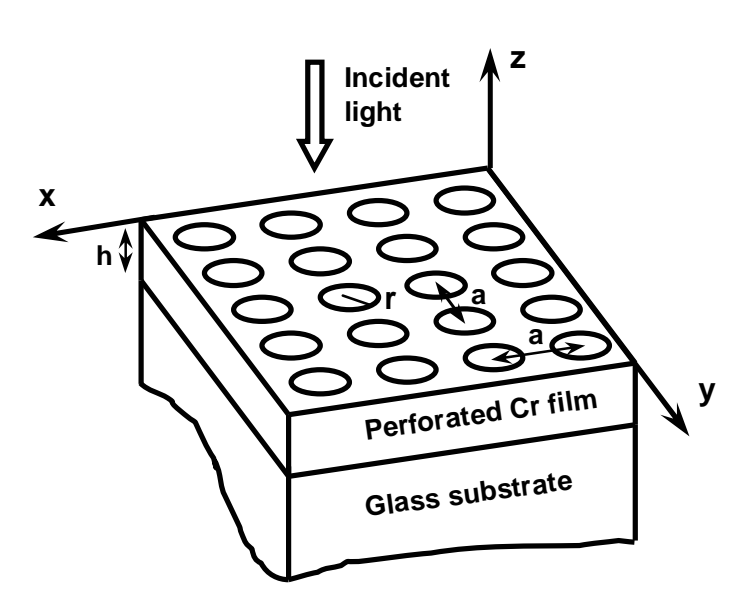




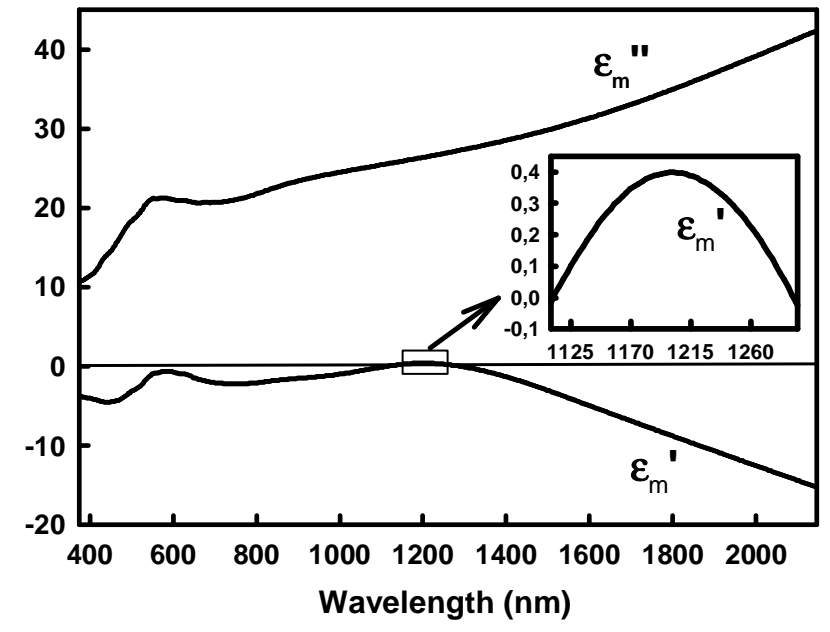




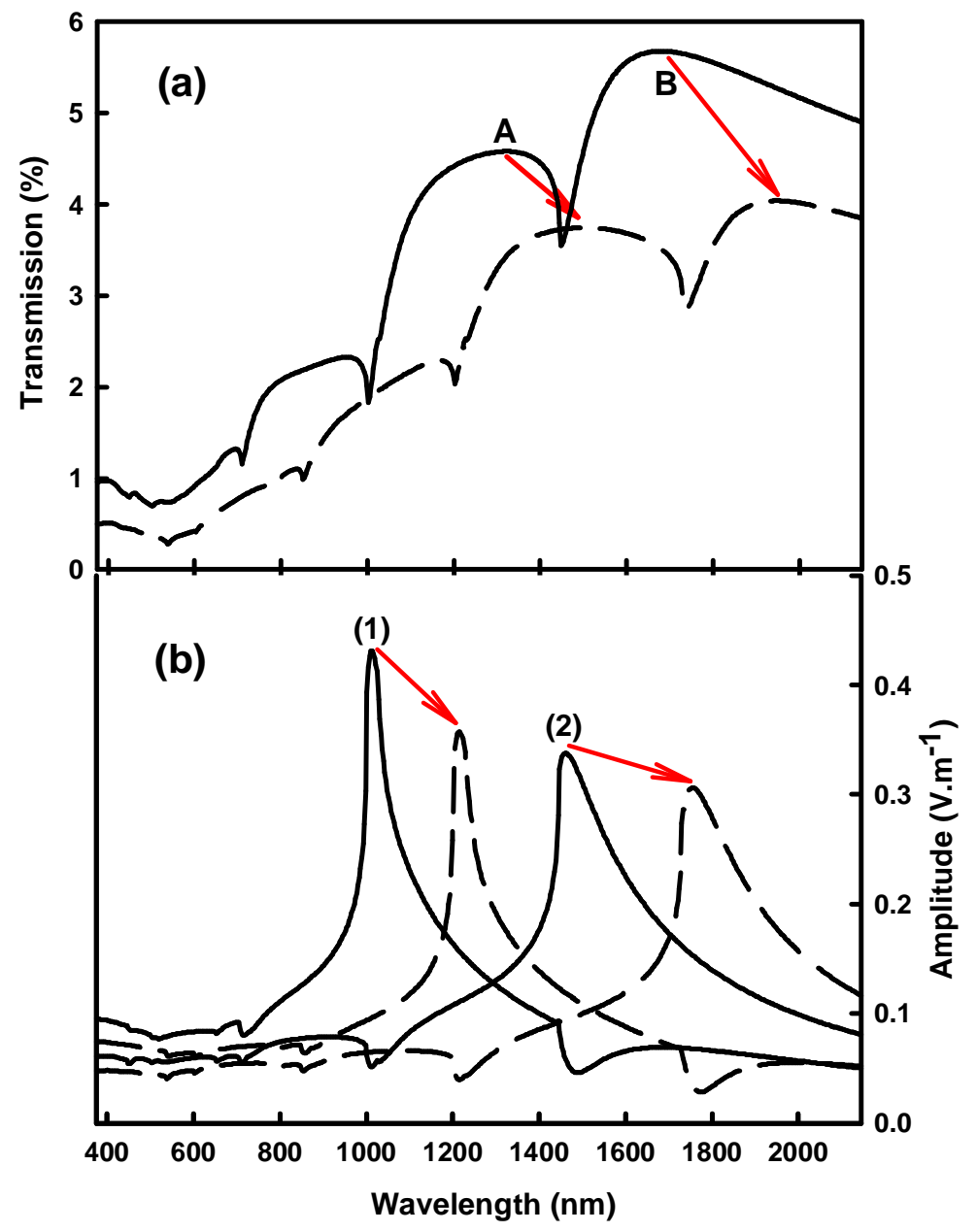




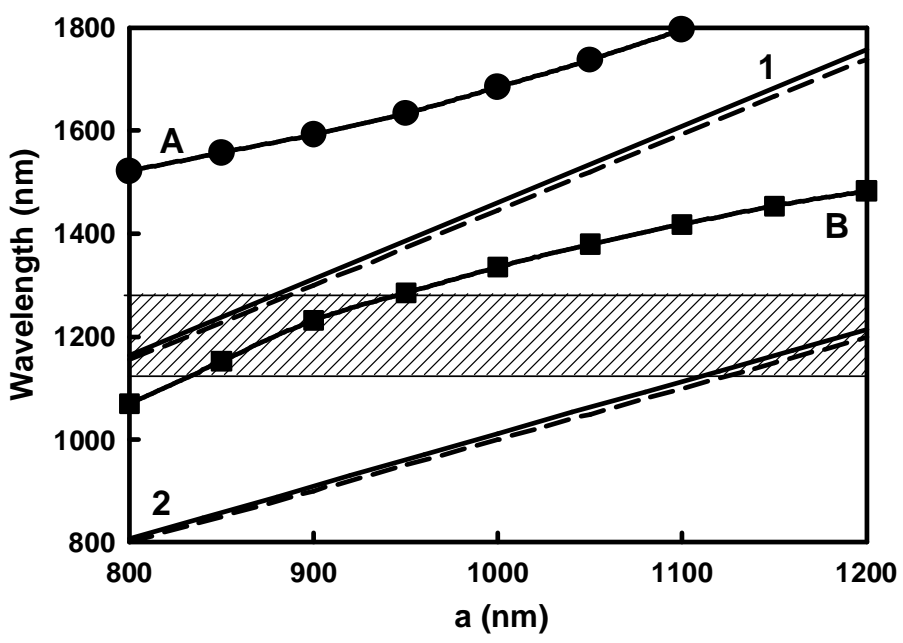

